\title{
Eficacy of Cryotherapy in the Prevention of Oral Mucosistis in Adult Patients with Chemotherapy
}

\author{
Ángel López-González ${ }^{1,2}$, Marta García-Quintanilla ${ }^{1}$, Carmen María Guerrero-Agenjo ${ }^{3}$, Jaime López Tendero ${ }^{3}$, \\ Isabel María Guisado-Requena ${ }^{1,2, * \mathbb{D}}$ and Joseba Rabanales-Sotos ${ }^{1,2}$ (D) \\ 1 Department of Nursing, Physiotherapy and Occupational Therapy, Facultad de Enfermería, Universidad de \\ Castilla-La Mancha, Campus Univesitario s/n, 02071 Albacete, Spain; angel.lopez@uclm.es (Á.L.-G.); \\ gmartaq97@gmail.com (M.G.-Q.); joseba.rabanales@uclm.es (J.R.-S.) \\ 2 Group of Preventive Activities in the University Health Sciences Setting, Universidad de Castilla-La \\ Mancha/UCLM, 13001 Ciudad Real, Spain \\ 3 Castilla-La Mancha Health Service (Servicio de Salud de Castilla-La Mancha/SESCAM), Universidad de \\ Castilla-La Mancha/UCLM, 02071 Albacete, Spain; carmenmga@hotmail.com (C.M.G.-A.); \\ leman_1988@hotmail.com (J.L.T.) \\ * Correspondence: IsabelM.Guisado@uclm.es; Tel.: +34-967-599-200 (ext. 2735)
}

\section{check for} updates

Citation: López-González, Á.; García-Quintanilla, M.; GuerreroAgenjo, C.M.; Tendero, J.L.; GuisadoRequena, I.M.; Rabanales-Sotos, J. Eficacy of Cryotherapy in the Prevention of Oral Mucosistis in Adult Patients with Chemotherapy. Int. J. Environ. Res. Public Health 2021, 18, 994. https://doi.org/10.3390/ ijerph18030994

Academic Editors: Paul B. Tchounwou and Takaaki Tomofuji Received: 24 November 2020

Accepted: 19 January 2021

Published: 23 January 2021

Publisher's Note: MDPI stays neutral with regard to jurisdictional claims in published maps and institutional affiliations.

Copyright: (c) 2021 by the authors. Licensee MDPI, Basel, Switzerland. This article is an open access article distributed under the terms and conditions of the Creative Commons Attribution (CC BY) license (https:// creativecommons.org/licenses/by/ $4.0 /)$.

\begin{abstract}
Oral mucositis (OM) is a common side effect of cancer therapies. It causes ulcerative, painful lesions in the oral cavity that can provoke malnutrition, increased risk of infection, longer hospital stays, and seriously affect the quality of life. Cooling the mucosa with oral cryotherapy (OC) during and/or after chemotherapy is the most accessible and tolerable intervention available. The aim of this study is to define the efficacy of OC for preventing OM induced by chemotherapy/radiotherapy in adult patients with cancer. Secondary endpoints include associated problems as pain. A systematic search was performed using the Pubmed, WOS (Web of Science), Cochrane Library, CINAHL, and BVS databases for articles published up to 2010. After inclusion and exclusion criteria were applied, a total of eight articles were analyzed in this review. In seven of the eight articles, the incidence of $\mathrm{OM}$ of all grades was significantly lower in the OC group compared with the no-OC group. Use of opioids and level of pain were also significantly reduced. OC is an effective intervention to reduce the incidence of OM induced by chemotherapy as well as the associated severity and pain. Based on these results, OC with only water or with chamomile, associated or not with other mouthwash therapies, is an effective intervention to reduce the incidence of OM induced by chemotherapy as well as the associated severity and pain.
\end{abstract}

Keywords: oral mucositis; patients' cancer; oral mucositis prevention; oral cryotherapy

\section{Introduction}

Oral mucositis (OM) is one of the most frequent complications suffered by patients affected by various types of cancer who are treated with chemotherapy, radiotherapy, or a combination of both. OM is defined as an alteration of the oral mucosa that causes inflammation and ulcerative lesions [1-3].

OM occurs in $20-40 \%$ of patients treated with conventional chemotherapy, in $80 \%$ of patients treated with high-dose chemotherapy prior to autologous bone marrow transplantation (HSCT), and even in almost all patients with head and neck cancer treated with radiotherapy combined with chemotherapy [1-5].

The physiopathogenesis of OM is a "cascade" process; the currently most accepted explanatory model describes it in five phases. In the first phase or "initiation phase", cytotoxic agents or radiation cause DNA breakage of the epithelial basal cells, causing cell damage. This damage then leads to increased production of proinflammatory cytokines, which, in turn, induce cell death (transcription phase). At this point, the initial response to cell damage is magnified, and a positive feedback process is created in which more and 
more tissue is damaged (amplification phase). All this leads to the formation of ulcerative wounds and bacterial colonization, which overstimulates the inflammatory response, increasing the damage of the oral mucosa (ulceration phase). The process concludes in the healing phase after the treatment action is completed, the cells regenerate, and normal microbiota is restored [1-6].

The scale proposed by the WHO classifies it in five degrees: Grade 0, incipient and asymptomatic lesions; Grade I, oral soreness and erythema; Grade II, oral erythema, ulcers, solid diet tolerated; Grade III, oral ulcers, liquid diet only; Grade IV, oral alimentation impossible. On this scale, Grades III and IV correspond to severe OM [3].

The first manifestation of OM usually appears between the third and seventh day at the beginning of treatment; there is localized or generalized erythema, and this may be accompanied by stinging, the most affected tissues being the soft palate, the lateral edges of the tongue, the buccal mucosa, the tonsils, and the pharyngeal wall. In patients receiving chemotherapy for the treatment of solid tumors, OM may not progress to more severe stages due to the use of lower doses of chemotherapy/radiation. However, a large number of patients evolve and develop one or more ulcers, this being the period in which the patient experiences greater pain and discomfort. In the majority of those affected, it evolves into mucosal ulceration, this being the period in which the patient experiences the most pain and discomfort [7-9].

Severe OM is characterized by intense pain associated with ulcerative lesions as the main complication, originating a difficulty in feeding and a decrease in caloric contribution $[2,6,7]$. The inadequate nutrition will affect the immune system, and the ulcers are usually colonized by oral bacterial flora, very often by the herpes simplex and candida viruses. In patients who are immunosuppressed by chemotherapy, these lesions can be a route of entry for systemic infections, causing sepsis and potentially posing a danger to life [7]. It may become necessary to reduce and even suspend the doses of chemo/radiotherapy, with the worsening of the prognosis of the disease and the patient's quality of life $[6,7,10]$.

The main factors related to its appearance are related to the type and dose of cytostatic agents used. Regarding the type of agent, the most important are the antimetabolites (methotrexate, fluoronacil, cytarabine), which affect DNA synthesis and are associated with about $40-60 \%$ of OM incidences. In terms of dosage, it has been shown that the risk of suffering from OM increases with the intensity of the treatment, whatever the drugs chosen. Moreover, the planning of the cycles, their duration, the route of administration, localized radiotherapy in the head and neck, and the combination of chemo/radiotherapy are risk factors. Therefore, almost all patients who undergo an HSCT develop OM induced by the conditioning treatments $[2,6,9]$.

Other possible factors such as age, type of tumor, poor oral health before and after treatment, malnutrition, alterations in the production and composition of saliva, and liver and kidney function could not be demonstrated in the appearance of OM [8,9].

The objectives of OM treatment are to prevent or reduce the severity of the lesions and to manage the associated symptoms, allowing the continuity of the cancer therapy [1,2].

The Multinational Association of Supportive Care and the International Society of Oral Oncology (MASC/ISOO) (Aurora, Ontario, Canada) have proposed, among the treatment methods for $\mathrm{OM}$, basic oral care, application of growth factors and cytosines, anti-inflammatory agents, antimicrobials, protective agents, anesthetics and analgesics, laser and other phototherapies, natural agents and the application of oral cryotherapy (OC) $[1,2,11]$.

OC consists of the local cooling of oral mucosal tissues using small pieces or sheets of ice, which, in the case of administration of chemotherapeutic agents, will produce vasoconstriction and decrease the distribution of the drug by the cells of the oral cavity. In the case of radiation therapy, the cold decreases the inflammation of the tissue reached by the radiation, which, in turn, reduces cell damage and prevents ulcerations [12-14]. 
OC is a normally well-tolerated intervention, with headache, tooth sensitivity, and numbness of the mouth being the most common side effects. In addition, it is the most accessible and efficient technique of all the proposals $[15,16]$.

\section{Objectives: Peak Question}

Due to the controversy about whether or not OC therapy is beneficial for patients with $\mathrm{OM}$, this review aims to find out whether $\mathrm{OC}$ is effective in preventing severe $\mathrm{OM}$ and its influence on the onset or evolution of pain in adult cancer patients treated with chemotherapy.

\section{Materials and Methods}

\subsection{Information Sources}

The following review was conducted through a literature search that began in October 2019 and ended in January 2020 in the following health-related databases: Pubmed, WOS (Web of Science), Cochrane Library, CINHAL (Cumulative Index to Nursing and Allied Health Literature), and Biblioteca Virtual en Salud (BVS).

\subsection{Search Strategy}

The search strategy was based on the keyword search string in the MeSH/DeCS descriptors of the databases mentioned above. The string was filled in with the Boolean operators AND and OR.

Table 1 shows the PICO criteria used and how each part of the search string belongs to each PICO criteria, following the appropriate structure with its corresponding keywords.

Table 1. PICO criterion.

\begin{tabular}{cc}
\hline Criterion (PICO) & Keywords \\
\hline Population (P) & $\begin{array}{c}\text { ("patients' cancer") and ("oral mucositis") and } \\
\text { ("chemotherapy") and ("radiotherapy") }\end{array}$ \\
\hline Intervention (I) & oral cryotherapy \\
\hline Outcome (O) & OM prevention OR patient's benefits \\
\hline
\end{tabular}

\subsection{Inclusion Criteria}

The inclusion criteria included publication within the last 10 years (1 February 2010 through to 31 January 2020) and an adult patient population in which OC has been used to prevent or treat $\mathrm{OM}$ that appeared because of the treatment of their cancer with chemotherapy and/or radiation therapy.

The exclusion criteria were animal experimentation and OM produced by causes other than cancer treatment with chemotherapy or radiotherapy. Systematic reviews were excluded due to a lack of scientific quality.

\subsection{Selection of Studies and Collection of Data}

After an exhaustive search, a total of 368 results were obtained, of which 326 were eliminated by title and summary, leaving a total of 42 (Figure 1). Twenty-one duplicates were removed. The review was conducted independently by two researchers using the inclusion and exclusion criteria of this review.

Thus, a total of 21 articles were chosen for the literature review. However, 13 of them were discarded for not meeting the established inclusion criteria.

The results were structured under a standardized register using author, year, type of study, objective, randomization, blinded, country, duration of the study, patients who developed OM as a complication of cancer treated with chemotherapy and/or radiotherapy, and intervention. 


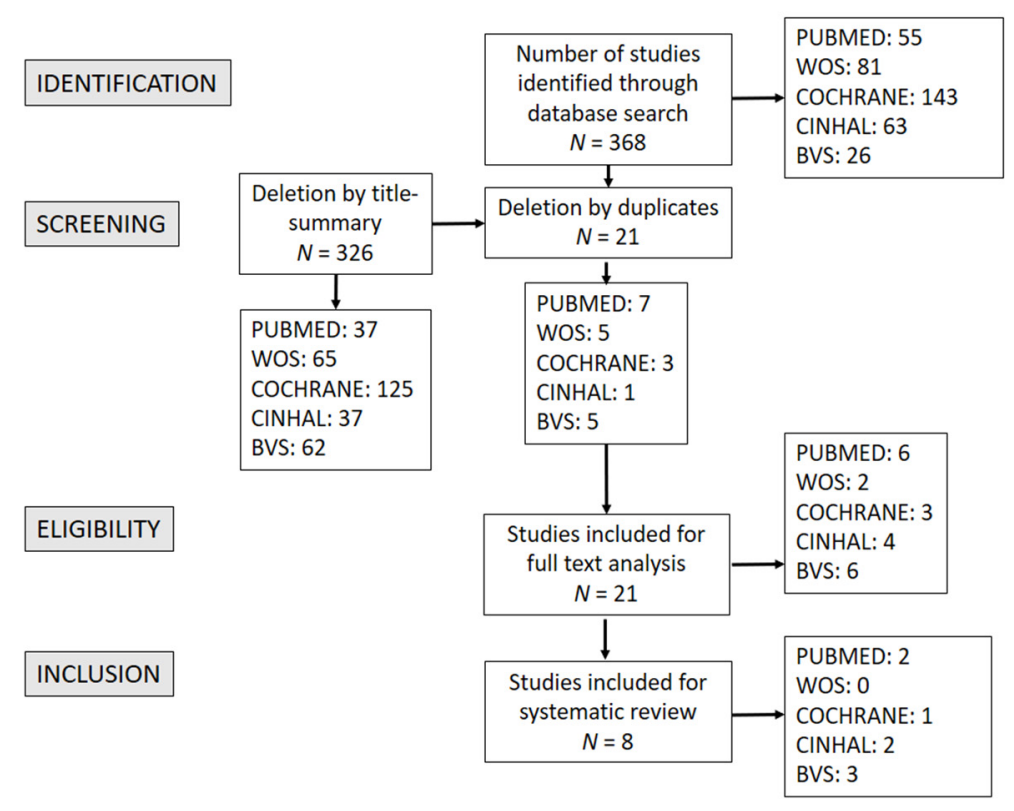

Figure 1. Prisma flow chart.

\subsection{Assessment of the Quality of Studies: Detection of Possible Bias}

Rating scales were made to evaluate the quality of the studies. The PEDRO scale (11 items) was used for randomized controlled trials (RCTs) [17]. The selection criteria were taken into account if the study population was randomized and the study was blinded for the intervention. The groups were similar at the start of the study, and all subjects, therapists or those who collected the data, and evaluators were blinded as to whether there was a high proportion of population lost during the study.

In three observational case-control studies, the Newcastle-Ottawa scale for casecontrol was applied [18].

These scales were applied by one reviewer and analyzed by a second reviewer to detect possible biases.

All the articles included are characterized by a low risk of bias, according to the scales carried out.

\subsection{Analysis of Data and Levels of Evidence}

The degree of evidence depends on factors such as the type of study and the methodological quality. To evaluate the level of evidence, a qualitative assessment was carried out using the scale of the Agéncia dÁvaluació de Tecnología Médica [19]. According to this scale, all the selected RCTs are of high relevance since, as mentioned in the previous section, all of them have a low risk of bias and are observational case-control studies.

\section{Results}

After an exhaustive search of the different databases, a total of 368 results were obtained, of which 8 were finally selected to carry out this literature review. These articles were selected using the inclusion criteria. The populations studied come from Italy, Brazil, Turkey, Malaysia, Spain, Japan, and Canada.

The studies reviewed included between 38 and 140 participants, with a greater predominance of men in 7 of the eight articles reviewed [16,17,19-23]. All of them included adult patients, ranging in age from 18 to over 70 years. Tables 2 and 3 show the characteristics and results of the selected studies. 
Table 2. Description of the type of study, intervention, sample size, and year of the selected studies.

Author-Year

Marchesi F. et al., 2017 [20]
Randomization method: it is based on two groups (experimental and control). Place: Hospital Sant'Eugenio in Roma (Italy). Participants were not blinded. All patients signed a written informed consent, and the study was approved by the Ethical Committee, in compliance with the Helsinki declaration.

\section{Type of Intervention}

Sample Size

From October 2013 to January 2016, patients were

enrolled in this study.

CG: oral standard care.

EG: after IV melphalan administration, on day-2, patients in EG received ice chips with rounded

corners in their mouth during chemotherapy infusion. When the ice melted, it was immediately replaced.

$\mathrm{OM}$ was assessed daily using the National Cancer Institute Common Toxicity Criteria version 4.02 .

Patient pain due to $\mathrm{OM}$ was monitored daily using the numerical rating scale. In case of severe and uncontrolled pain, IV opioids were administered.

All patients (EG and CG) underwent uniform anti-infectious and support therapy (ciprofloxacin, cotrimoxazole, and valaciclovir). All patients (EG and CG) underwent mouth rinses with oral

nystatin-based protocols three times daily.

Patients were hospitalized and remained as inpatients for the duration of the study.

No data on how long patients received chemotherapy or how long after therapy when some degree of OM appeared.
$N=72$ (36 EG, 36 CG), 46 males, 26 females. Inclusion criteria: Patients aged $>18$ years, with multiple myeloma undergoing HSCT after being treated with high doses of melphalan.

Exclusion criteria: Patients who had experienced previous episodes of OM or with previous exposure to chemotherapy or neck/head radiotherapy were excluded.

Characteristics: age (EG: $58 \pm 13.5$; CG: $56 \pm 17$ ) 
Table 2. Cont.

Author-Year $\quad$ Type of Study

Diniz et al., 2016 [21]
Randomization method: it is based on two groups (experimental and control). Place: Hospital Center of High Complexity Oncology del Hospital Universitario en Brasilia (Brasil). Only the doctor was blinded to randomization. Patients were not possible to blind. All patients provided written consent prior to starting the procedures. The study was approved by the Committee on Ethics Research of the School of Health Sciences of the University of Brasília.

\section{Type of Intervention}

Sample Size

Between March 2012 and March 2015, patients were invited to participate in the study. The study interventions were performed only during the first 5 days of the first cycle of chemotherapy. Patients who agreed to participate in the study watched a video explaining how to perform oral hygiene and received an oral

hygiene kit (toothbrush, nonabrasive toothpaste, and dental floss). During chemotherapy

treatment, the following were

administered prophylactically:

CG: OC with only water was

administered prophylactically.

EG: Patients in the control group received a cup of ice chips made with pure water, while patients in

the chamomile group received a cup of ice chips made with chamomile infusion at $2.5 \%$. Both groups were instructed to swish the ice around in their oral cavity for at least $30 \mathrm{~min}$, starting $5 \mathrm{~min}$ before the chemotherapy infusion. During the intervention, patients were asked to fill a questionnaire about ice taste, discomfort, and pain regarding cryotherapy. A doctor evaluated the oral mucosa on days 8,15 , and 22 after the first chemotherapy infusion.

Patients underwent four to six courses of chemotherapy, each consisting of five consecutive days of chemotherapy infusion, followed by 21 days of rest. The study interventions were performed only during the 5 days of the first course.

No data showing how long after therapy before some degree of OM appeared.
$N=38$ (20 EG-11 males, 9 females, 18 CG -9 males, 9 females).

Inclusion criteria: elderly patients with gastric or colorectal cancer who received ambulatory IV chemotherapy for the first time (5-fluorouracil and leucovorin). Intact and healthy oral mucosa, without dental problems, and without a history of

hypersensibility or adverse reaction to chamomile or any plant of the Asteraceae or Compositae family. Characteristics: age EG: 54.7 (SD 8.15); CG: 55.2 (SD 9.5). 
Table 2. Cont.

Author-Year

Type of Study

Randomization method: it is based on three groups ( 2 experimental and 1 control).

Place: Erzurum Ataturk University Research and Application Hospital in Erzurum (Turkey).

Participants were not blinded. Oral consent was obtained from the patients for their participation in the study before the questionnaire forms were administered. The patients were also informed verbally about the study. Participation was voluntary, and the patients could withdraw from the study at any time without giving a reason.

Approval was obtained from the Ethics Committee of the Medical Faculty, Ataturk University.

\section{Type of Intervention}

Sample Size

The observation period was 15 days for each participant.

During chemotherapy treatment, the following were administered prophylactically:

EG1: received chlorhexidine mouthwash. EG2: OC with only water was
administered prophylactically.

$$
\text { CG: standard oral care. }
$$

EG1 data were collected from patients in the study groups who had oral care with chlorhexidine twice a day; EG2 had cryotherapy once a day. Chlorhexidine mouthwash was applied six times a day to patients with Grade III oral mucositis, and it was applied eight times a day to patients with Grade IV oral mucositis. Tooth brushing is not recommended to patients with Grade III and IV oral mucositis because of possible ulcerations due to physical irritation. CG data were collected from patients who followed standard oral care protocol (washing with plenty of water mouthwash).

The duration of the patient's disease was 4-9 or more months, and the duration of cancer therapy was 1-9 months.

No data showing how long patients received

chemotherapy or how longafter chemotherapy before some degree of OM appeared.
$N=90$ (30 EG1, 30 EG2, 30 CG).

Inclusion criteria: All subjects had Grade III-IV oral mucositis due to chemotherapy received for various types of cancer, and all of them were unable to take food orally.

Exclusion criteria were not named. 
Table 2. Cont.

Author-Year Type of Study

Randomization method: it is based on three groups ( 2 experimental and 1 control).

Svanberg et al., 2015 [23] Place: Akademiska University Hospital in Uppsala (Sweden). Participants were not blinded. The study was approved by the regional Research Ethics Committee.

\section{Type of Intervention}

Sample Size

From September 2010 and October 2011, patients were enrolled in this study.

During chemotherapy treatment, the following were administered prophylactically:

CG: OC with only water was

administered prophylactically.

EG: OC + Caphosol ${ }^{\circledR}$ was administered

prophylactically. OC was given in the form of ice

cubes or crushed ice to be kept in the mouth

during the actual infusion of the HSCT.

Thirty (30) mL Caphosol ${ }^{\circledR}$ was administrated for

rinsing the whole oral cavity four times/day,

starting prior to HSCT and ending on day 21.

All patients received intravenous conditioning

chemotherapy based on diagnosis. The average

duration of chemotherapy treatment, depending

on the type of cancer, was 4 days.

No data showing how long before some degree of

$$
\text { OM appeared. }
$$

During treatment with chemotherapy

(fluorounacil), the following was

administered prophylactically:

CG: standard care and rinses with sodium

Randomization method in two groups: experimentation and control.

Place: University of Malaya Medical Centre in Kuala Lumpur (Malaysia).

Idayu et al., 2018 [24]

Participants were not blinded. All patients

provided informed consent and were ensured of

the confidentiality of their participation. The

study was approved by the Ethical Committee. bicarbonate were administered prophylactically.

EG: were given ice chips to hold in their mouths for 30 min during chemotherapy administration (the ice chips were replenished as they melted, and patients were instructed to move the ice in an attempt to keep the entire oral cavity cold), followed by sodium bicarbonate mouthwash (three times daily) postchemotherapy until the next cycle.

No data showing the duration of the study, how long patients received chemotherapy, or how long after therapy before some degree of OM appeared
$N=40$ (20 EG-11 males, 9 females, 18 CG-9 males, 9 females).

Inclusion criteria: patients $>16$ years, with various types of cancer (acute myeloid leukemia, acute lymphoblastic leukemia, chronic lymphatic leukemia, chronic myeloid leukemia, chronic myelomonocytic leukemia, myelodysplastic syndrome), undergoing HSCT after being treated with chemotherapy and radiotherapy. All patients received intravenous conditioning chemotherapy and (when required) total body irradiation on the basis of diagnosis. Exclusion criteria were not named.

Characteristics: age between 17 and 67 years $(p>0.05$; EG: $50.4 \pm 10.6$; CG: $59.6 \pm 13.2$ )

\section{$N=80$ (40 EG-22 men, 18 women, 40 CG -23 men,} 17 women)

Inclusion criteria: patients $>20$ years old with colorectal cancer, scheduled for fluorouracil-based chemotherapy at their first cycle of chemotherapy. According to the Eilers scale (1988), only patients with scores ranging from 1-8 were recruited into the study.

Characteristics: age: between 20 and 60 years $($ mean $=48.4, \mathrm{SD}=9.2 ; p>0.05)$. 
Table 2. Cont.

Author-Year Type of Study

Batlle et al., 2014 [25]

Retrospective cohort study. Randomization method in two groups: experimentation and control.

Location: not specified.
Type of Intervention

Sample Size

From August 2006 to July 2011, 134 consecutive patients were enrolled in the study.

All participants underwent an oral care protocol consisting of a sodium bicarbonate mouthwash from day 7 of HSCT to hospital discharge.

CG: standard care and rinses with sodium bicarbonate were administered prophylactically. EG: OC consisted of ice chips sucked on before infusion (10 $\mathrm{min})$, during infusion (15 $\mathrm{min})$, and after chemotherapy administration for a total of

$40 \mathrm{~min}$. and then mouthwashes with sodium bicarbonate up to hospital discharge.

The mean time to the initiation and the duration of OM did not differ among groups (2.50 and 9.20 days, respectively).

No data on how long the patients received chemotherapy.

From March 2011 and July 2016, patients were enrolled in this study.

Retrospective cohort study.

Randomization method in two groups: experimentation and control.

Place: Department of Gastroenterological Surgery, Kanazawa University (Japan).

Okamoto et al., 2019 [26]

All procedures followed were in accordance with the ethical standards of the responsible committee

on human experimentation and the Helsinki

Declaration of 1964 and later versions. Informed consent or a substitute for it was obtained from all patients included in the study.

$$
\text { CG: did not receive OC. }
$$

EG: OC performed routinely for patients

receiving chemotherapy. The patients were instructed to suck continuously on several pieces of ice from $10 \mathrm{~min}$ before until after the end of the chemotherapy infusion.

The chemotherapy (cisplatin, 5-fluorouracil) was administered for $1-5$ days, repeated every

$$
4 \text { weeks. }
$$

No data showing how long before some degree of
$N=134$ (66 EG-47 men, 19 women, 68 CG-39 men, 29 women $)(p=0.094)$.

Inclusion criteria: patients $>20$ years old, with hematological cancer treated with chemotherapy (high-dose melphalan (HDmel)) and HSCT.

Characteristics: age between 23 and 70 years old. EG: 56 (23-69), CG: 55 (25-70) $(p>0.05)$
$N=72$ (58 EG—50 men, 8 women; CG 14-12 men, 2 women) $(p>0.999)$

Inclusion criteria: patients with primary esophagea cancer staged according to the 7th edition of the

tumor-node-metastasis (TNM) classification of malignant tumors and treated with chemotherapy prior to surgery.

Characteristics: age between 57 and 71 years old. EG: $64.0(57.0-69.0)$.

CG: 64.5 (57.3-70.5) $(p>0.05)$. OM appeared. 
Table 2. Cont.

Author-Year $\quad$ Type of Study

Chen et al., 2017 [27]
Retrospective cohort study.

Randomization method in two groups:

experimentation and control.

Place: Victoria Hospital, London Health

Sciences Center (UK).

Participants were not blinded.

The study protocol was approved by the Office of

Research Ethics at the University of Western

Ontario, Lawson Health Research Institute. Due

to the retrospective nature of the study, no patient consent was required.

\section{Type of Intervention}

Sample Size

The study examined patients over the span of seven years, from 2006 to 2013.

Medical charts of consecutive patients with

multiple myeloma undergoing autologous HSCT,

admitted over the period of 2006 to 2013, were reviewed.

Two groups of patients were compared in this study analysis:

CG: patients treated with chemotherapy between 2007 and 2009 who did not receive OC until 2010. EG: OC performed routinely for patients receiving chemotherapy from 2010 to 2013.

Patients were instructed to hold ice chips in their mouth for $5 \mathrm{~min}$ prior to high-dose melphalan infusion, during the 30-min infusion, and $30 \mathrm{~min}$ after completion of the infusion.

The conditioning regimen of chemotherapy (high-dose melphalan) was administered 2 days before the transplant date.

No data showing how long before some degree of $\mathrm{OM}$ appeared. The duration of OM in mean days was 10.1-7.8 (SD $4.9 \pm 6.2$, respectively).
$N=140$ (70 EG—56 men, 14 women; CG 70-38 men, 22 women) $(p=0.01)$.

Inclusion criteria: patients $>18$ years old, with lymphoma and multiple myeloma, receiving high-dose chemotherapy (mephalan) for HSCT.

Characteristics: age between 57 and 71 years old EG: $53.5( \pm 7.5)$,; CG: $56.5( \pm 7.3)(p=0.02)$. 
Table 3. Description of the results, conclusions, quality, and year of the selected studies.

Author-Year

Marchesi F et al., 2017 [20]

Diniz et al., 2016 [21]

EG patients never presented OM $\geq$ Grade $2(p>0.005)$.

OM appearance in any degree: EG: 30\%; CG: 50\% $(p=0.01)$

Mouth pain: EG had less mouth pain than CG $(p>0.05)$.

Oral nutrition transition time in days (mean $\pm \mathrm{SD}$ )

EG1: $8.53 \pm 1.04$. There was a statistical difference between experimental group 1 and the control group $(p<0.01)$.

Erden et al., 2017 [22] EG2: $12.13 \pm 1.81$. There was no statistical difference between experimental group 2 and the control group $(p>0.05)$.

CG: $13.53 \pm 1.69$. There was no statistical difference between experimental group 2 and the control group $(p>0.05)$.

\section{Conclusions}

Quality

The study provided relevant data to support

OC during HDM administration as the standard

of care in preventing $\mathrm{OM}$ in myeloma patients undergoing HSCT.

The infusion of OC with chamomile reduces the appearance of OM compared to OC with water alone; it also reduces mucosal pain.

The occurrence of OM was lower in patients who used OC made with chamomile infusion than in patients who used OC made only with water. When compared to the control group, the chamomile group presented less mouth pain and had no ulcerations. OC was well tolerated by both groups, and no toxicity was identified.

The analysis of this study showed that the

transition time of oral nutrition of the patients in the experimental group that applied

chlorhexidine was shorter than the transition time of oral nutrition of the patients of the group that applied OC; in both experimental groups (EG1 and EG2), transitional time was shorter than the control group. Parallel to this finding, it was found that the degree of $\mathrm{OM}$ was reduced. According to this result, using chlorhexidine or OC mouthwash for the prevention and

treatment of oral mucosis should be offered.

There is no difference between EG and CG in the degree of OM at day 21 of treatment ( 2.45 vs. 2.30 on average, according to the WHO scale; $p>0.05$ ).

Svanberg et al., 2015 [23]
The perception of oral pain in both groups, assessed with the visual analog scale, showed no difference ( 3.55 vs. 2.7 on the visual analog scale; $p>0.05$ ).
No additional significant effect of combining

Caphosol ${ }^{\circledR}$ with OC in the prevention and treatment of OM. 
Table 3. Cont.

Author-Year Results

\section{Conclusions}

Quality

OC followed by bicarbonate-based mouthwash

could help prevent oral mucositis and pain.

This finding helps shed light on evidence

supporting the use of oral cryotherapy, which is

cost-effective and has few side effects, as a

preventive strategy. OC is easily implemented

in tandem with the use of a sodium bicarbonate

mouthwash. The potential benefit of

cryotherapy in the prevention of oral mucositis

and the associated pain appears to improve the quality of life of patients undergoing

fluorouracil-based chemotherapy.

The authors indicated although this was a nonrandomized study and the conditioning regimens were not homogeneous, OC reduced the severity of $\mathrm{OM}$ in patients treated with regimens compared with saline rinses.

Additionally, OC was cost-effective and well

tolerated by the patients. In summary, OC

represents an effective and inexpensive

supportive measure to prevent OM induced by HDmel-based regimens.

$8 / 11$

\begin{abstract}
EG: $44 \%$ vs. CG $82 \%(p<0.001)$.
\end{abstract}
EG: $15 \%$ of OM Grades III and IV:
Opiates were required in EG: $10 \%$ and CG: $15 \%(p=0.305)$.
OC may be a useful prophylactic approach for

chemotherapy-induced OM in patients with esophageal cancer.

OC protocol implemented at HSCT resulted in significantly lower incidences

and severity of oral mucositis. The mean

duration of oral mucositis experienced by

patients was shortened, and the need for the use

of parenteral narcotics was decreased as well.

These results provide evidence for the

continued use of oral cryotherapy, an

inexpensive and generally well-tolerated

practice, in patients receiving high-dose

melphalan for autologous HSCT.
The mean degree of OM in the CG vs. the EG was higher (2.5 vs. $2 ; p=0.03$. CG $(44.2 \%), p=0.02$. 
In four of the studies, patients underwent HSCT with high-dose chemotherapy [20,23-25] while in the other four studies, the patients suffered from cancer of the digestive system (colorectal, stomach, esophagus, and pancreas) $[21,22,24,26]$. The study periods were from four months to seven years in duration. All selected studies focused on OM produced by chemotherapy treatments. Chemotherapy regimens were mainly based on melphalan in cases of HSCT [20,23,25,27] and fluoronacil in cases of digestive cancer $[21,24,26]$. Regarding the design of the studies, in all of them, the experimental group (EG), formed by the patients who were treated with OC, was compared with a control group (CG). In four of the articles, patients who received standard oral hygiene were assigned the CG [20,25-27]. In another three articles, OC was compared to the effects of other types of treatments for OM applied to the CG, such as chlorhexidine rinses [22], bicarbonate rinses [24], and the use of Caphosol ${ }^{\circledR}$ (EUSA Pharma (Europe) Limited - Stevenage, UK) [23]. In addition, a variant of OC made with chamomile infusion was analyzed in the remaining study [21].

\section{Discussion}

$\mathrm{OM}$ is one of the adverse effects of chemotherapy and radiotherapy that mostly worsens the quality of life of cancer patients in addition to increasing hospitalizations and financial expenses. There are many treatments that have tried to reduce both its incidence and its severity, including OC.

The present literature review confirms the benefits of $\mathrm{OC}$ in the control of OM produced as an adverse effect of chemotherapy treatment in cancer patients, as well as being cheaper, more accessible, and better tolerated by patients.

\subsection{OC in OM Prevention}

The effectiveness of $O C$ was demonstrated in six of the articles, where the incidence and severity of OM were found to be significantly lower in the EG than in the CG, to which it was not applied [20,23-27]. Only one of them proposed the use of chlorhexidine rinses instead of OC to decrease the incidence of OM and to facilitate an oral diet [22]. In the remaining study, it was proposed to replace conventional ice with ice cubes made with chamomile infusion since the incidence of $\mathrm{OM}$ and the pain perceived by the patients in that EG were lower [21].

Additionally, the occurrence of severe OM was studied in five articles, and in all of them, a significant reduction was observed in the groups to which OM was applied, even to the point of never occurring $[20,24-27]$.

\subsection{Influence of OC on the Occurrence of Pain in Adult Cancer Patients Treated with Chemotherapy}

Pain is a variable that was taken into account in six of the studies, measured based on the need for the use of opioids or through rating scales $[20,21,23-25,27]$. In the first case, Marchesi et al. and Chen et al. reported that the administration of opioid analgesics was significantly lower in the EG [20,27]; however, Batlle et al. found no difference between the EG and the CG [25]. In terms of direct pain assessment, in two of the articles, patients treated with OC expressed lower scores than those who were not [21,24]. Furthermore, in the study by Svanberg et al., it was observed that the association of OC $+\mathrm{Caphosol}^{\circledR}$ offered no additional effect on pain compared to using OC alone [23].

Finally, seven of the eight selected articles support the use of OC, including a camomileinfused variant, as an effective measure to decrease its incidence and severity $[20,21,23-27]$. Finally, in all of them, the treatment was well-tolerated in its great majority and was without adverse effects.

\subsection{Strengths and Limitations}

The present review presents some limitations, such as a sample that does not allow the generalization of the results found and the variability of the types of OC used.

Among the strengths is the recent research included (from the last 10 years), so the information is current. In addition, the results of the studies are similar, and the quality of 
all of them is high. Moreover, the great variability of the countries where the studies have been carried out provides a global vision.

Due to the scarcity of articles on the effect of $\mathrm{OC}$ in the prevention of OM and its main complications, such as pain, more research should be carried out with larger population samples to obtain conclusive data.

\subsection{Implications for Clinical Practice}

$\mathrm{OC}$ is an effective treatment to decrease the incidence of $\mathrm{OM}$ in patients receiving chemotherapy/radiotherapy. It is also effective in preventing the progression of more severe phases of ulceration.

OC decreases pain in patients with OM that has developed as a result of their cancer treatment with chemotherapy/radiotherapy.

It is a very safe therapeutic option due to the great tolerance of the patients and the scarcity of adverse effects, besides being a very cheap and affordable resource for any institution.

\section{Conclusions}

After analyzing the selected articles, we conclude that OC is an effective treatment to prevent the appearance of oral $\mathrm{OM}$ in patients who are being treated for cancer with chemotherapy/radiotherapy. OC application avoids the worsening of OM to a more serious phase of ulceration and alleviates its main symptom, pain.

OC is a therapeutic option that has been shown to be safe for patients, with a high tolerance level, given the scarcity of adverse effects. OC is a very economical resource that is affordable for any institution, showing itself as a highly efficient therapeutic option.

$\mathrm{OM}$ is a very widespread complication with a high impact on the quality of life of patients.

Author Contributions: Conceptualization, Á.L.-G. and M.G.-Q.; methodology, J.R.-S., Á.L.-G., and I.M.G.-R.; software, J.R.-S. and J.L.T.; validation, J.R.-S., J.L.T., C.M.G.-A., and A.L.-G.; formal analysis, Á.L.-G., I.M.G.-R., J.R.-S. and J.L.T.; investigation, J.R.-S., Á.L.-G., C.M.G.-A., and M.G.-Q.; resources, J.R.-S. and Á.L.-G.; data curation, J.R.-S., Á.L.-G., I.M.G.-R., and C.M.G.-A.; writing-original draft preparation, J.R.-S., Á.L.-G., and M.G.-Q.; writing-review and editing, J.R.-S., Á.L.-G., I.M.G.R., J.L.T., C.M.G.-A., and M.G.-Q.; visualization, J.R.-S., Á.L.-G., J.L.T., C.M.G.-A., and M.G.-Q.; supervision, J.R.-S., Á.L.-G., and I.M.G.-R.; project administration, J.R.-S. and Á.L.-G.; funding acquisition, J.R.-S., Á.L.-G., M.G.-Q., and I.M.G.-R. All authors have read and agreed to the published version of the manuscript.

Funding: This research was funded by a grant from the European Regional Development Fund (ERDF; Fondo Europeo de Desarrollo Regional (FEDER; DOCM 19/02/20). The researcher Isabel María Guisado-Requena receives funding from the European Regional Development Fund (FEDER; 2018/11744).

Institutional Review Board Statement: Not applicable.

Informed Consent Statement: Not applicable.

Data Availability Statement: The study did not report any data.

Conflicts of Interest: The authors declare no conflict of interest.

\section{References}

1. Daugèlaitè, G.; Užkuraitytè, K.; Jagelavičienè, E.; Filipauskas, A. Prevention and Treatment of Chemotherapy and Radiotherapy Induced Oral Mucositis. Medicina 2019, 55, 25. [CrossRef] [PubMed]

2. Lalla, R.V.; Saunders, D.P.; Peterson, D.E. Chemotherapy or radiation-induced oral mucositis. Dent. Clin. N. Am. 2014, 58, 341-349. [CrossRef] [PubMed]

3. Alvariño-Martín, C.; Sarrión-Pérez, M.G. Prevention and treatment of oral mucositis in patients receiving chemotherapy. J. Clin. Exp. Dent. 2014, 6, e74-e80. [CrossRef] [PubMed]

4. Epstein, J.B.; Miaskowski, C. Oral Pain in the Cancer Patient. J. Natl. Cancer Inst. Monogr. 2019, 2019. [CrossRef] 
5. Fernández, M.H.; Villaverde, R.M.; Soto, M.Á.-M. Protocolo de manejo de la mucositis oral en el paciente oncológico. Rev. Educ. Super 2017, 12, 45-53.

6. Mallick, S.; Benson, R.; Rath, G.K. Radiation induced oral mucositis: A review of current literature on prevention and management. Eur. Arch. Otorhinolaryngol. 2016, 273, 2285-2293. [CrossRef]

7. Park, S.H.; Lee, H.S. Meta-analysis of oral cryotherapy in preventing oral mucositis associated with cancer therapy. Int. J. Nurs. Pract. 2019, 25, e12759. [CrossRef]

8. Al-Ansari, S.; Zecha, J.A.; Barasch, A.; de Lange, J.; Rozema, F.R.; Raber-Durlacher, J.E. Oral Mucositis Induced By Anticancer Therapies. Curr. Oral. Health Rep. 2015, 2, 202-211. [CrossRef]

9. Chaveli-López, B.; Bagán-Sebastián, J.V. Treatment of oral mucositis due to chemotherapy. J. Clin. Exp. Dent. 2016, 8, e201-e209. [CrossRef]

10. Bourdelin, M.; Daguindau, E.; Larosa, F.; Legrand, F.; Nerich, V.; Deconinck, E.; Limat, S. Mucositis after allogeneic stem cell transplantation: Risk factors, clinical consequences and prophylaxis. Pathol. Biol. 2015, 63, 106-110. [CrossRef]

11. Lalla, R.V.; Bowen, J.; Barasch, A.; Elting, L.; Epstein, J.; Keefe, D.M.; McGuire, D.B.; Migliorati, C.; Nicolatou-Galitis, O.; Peterson, D.E.; et al. MASCC/ISOO clinical practice guidelines for the management of mucositis secondary to cancer therapy. Cancer 2014, 120, 1453-1461. [CrossRef] [PubMed]

12. Peterson, D.E.; Ohrn, K.; Bowen, J.; Fliedner, M.; Lees, J.; Loprinzi, C.; Mori, T.; Osaguona, A.; Weikel, D.S.; Elad, S.; et al. Systematic review of oral cryotherapy for management of oral mucositis caused by cancer therapy. Support. Care Cancer 2013, 21, 327-332. [CrossRef] [PubMed]

13. Manzi, N.M.; Silveira, R.C.; dos Reis, P.E. Prophylaxis for mucositis induced by ambulatory chemotherapy: Systematic review. J. Adv. Nurs. 2016, 72, 735-746. [CrossRef] [PubMed]

14. Svanberg, A.; Ohrn, K.; Birgegård, G. Five-year follow-up of survival and relapse in patients who received cryotherapy during high-dose chemotherapy for stem cell transplantation shows no safety concerns. Eur. J. Cancer Care 2012, 21, 822-828. [CrossRef]

15. Wodzinski, A. Potential Benefits of Oral Cryotherapy for Chemotherapy-Induced Mucositis. Clin. J. Oncol. Nurs. 2016, 20, 462-465. [CrossRef]

16. Kadakia, K.C.; Rozell, S.A.; Butala, A.A.; Loprinzi, C.L. Supportive cryotherapy: A review from head to toe. J. Pain Symptom Manag. 2014, 47, 1100-1115. [CrossRef]

17. Cardoso-Ribero, C.; Gómez-Conesa, A.; Hidalgo-Montesinos, M. Metodología para la adaptación de instrumentos de evaluación. Fisioterapia 2011, 32, 264-270. [CrossRef]

18. Silva, F.C.; Arancibia, B.V.; Iop, R.R.; Gutierres Filho, P.J.B.; Silva, R. Escalas y listas de evaluación de la calidad de estudios científicos. Evaluation lists and escales for the quiality of scientific studies. Rev. Cuba. Inf. Cienc. Salud 2015, 24, 295-312.

19. Jovell, A.; Navarro-Rubio, M. Evaluación de la evidencia científica. Med. Clin. 1995, 105, 740-743.

20. Marchesi, F.; Tendas, A.; Giannarelli, D.; Viggiani, C.; Gumenyuk, S.; Renzi, D.; Franceschini, L.; Caffarella, G.; Rizzo, M.; Palombi, F.; et al. Cryotherapy reduces oral mucositis and febrile episodes in myeloma patients treated with high-dose melphalan and autologous stem cell transplant: A prospective, randomized study. Bone Marrow Transplant. 2017, 52, 154-156. [CrossRef]

21. Dos Reis, P.E.; Ciol, M.A.; de Melo, N.S.; Figueiredo, P.T.; Leite, A.F.; Manzi, N.M. Chamomile infusion cryotherapy to prevent oral mucositis induced by chemotherapy: A pilot study. Support. Care Cancer 2016, 24, 4393-4398. [CrossRef] [PubMed]

22. Erden, Y.; Ipekcoban, G. Comparison of efficacy of cryotherapy and chlorhexidine to oral nutrition transition time in chemotherapyinduced oral mucositis. Eur. J. Cancer Care 2017, 26. [CrossRef] [PubMed]

23. Svanberg, A.; Öhrn, K.; Birgegård, G. Caphosol $\left({ }^{\circledR}\right)$ mouthwash gives no additional protection against oral mucositis compared to cryotherapy alone in stem cell transplantation. A pilot study. Eur. J. Oncol. Nurs. 2015, 19, 50-53. [CrossRef] [PubMed]

24. Okamoto, K.; Ninomiya, I.; Yamaguchi, T.; Terai, S.; Nakanuma, S.; Kinoshita, J.; Makino, I.; Nakamura, K.; Miyashita, T.; Tajima, H.; et al. Oral cryotherapy for prophylaxis of oral mucositis caused by docetaxel, cisplatin, and fluorouracil chemotherapy for esophageal cancer. Esophagus 2019, 16, 207-213. [CrossRef] [PubMed]

25. Batlle, M.; Morgades, M.; Vives, S.; Ferrà, C.; Oriol, A.; Sancho, J.-M.; Xicoy, B.; Moreno, M.; Magallón, L.; Ribera, J.-M. Usefulness and safety of oral cryotherapy in the prevention of oral mucositis after conditioning regimens with high-dose melphalan for autologous stem cell transplantation for lymphoma and myeloma. Eur. J. Haematol. 2014, 93, 487-491. [CrossRef] [PubMed]

26. Nawi, R.I.M.; Chui, P.L.; Ishak, W.Z.W.; Chan, C.M.H. Oral Cryotherapy: Prevention of Oral Mucositis and Pain Among Patients with Colorectal Cancer Undergoing Chemotherapy. Clin. J. Oncol. Nurs. 2018, 22, 555-560. [CrossRef]

27. Chen, J.; Seabrook, J.; Fulford, A.; Rajakumar, I. Icing oral mucositis: Oral cryotherapy in multiple myeloma patients undergoing autologous hematopoietic stem cell transplant. J. Oncol. Pharm. Pract. 2017, 23, 116-120. [CrossRef] 